\title{
Long-term auditory follow-up in counteracting platinum-induced ototoxicity in paediatric oncology
}

\author{
ANNA FETONI ${ }^{1}$, eugenio de corso ${ }^{1}$, Francesca Brigato ${ }^{2}$, Daniela Lucidi $^{1}$, walter di nardo ${ }^{2}$, \\ EMANUELE SCARANO ${ }^{1}$, JACOPO GALLI ${ }^{2}$, and Antonio Ruggiero ${ }^{3}$ \\ ${ }^{1}$ Policlinico Universitario Agostino Gemelli \\ ${ }^{2}$ Universita Cattolica del Sacro Cuore Facolta di Medicina e Chirurgia \\ ${ }^{3}$ Fondazione Policlinico Universitario Gemelli IRCCS - Università Cattolica del Sacro \\ Cuore Roma
}

October 5, 2020

\begin{abstract}
Background. Irreversible bilateral sensorineural hearing loss is a common side effect of platinum compounds. Because of extended overall survival period of children, a prolonged hearing surveillance and management of hearing impairments are emerging concerns for pediatric oncology. Methods. In this retrospective observational study we enrolled 38 children out of 116 treated at our institution by chemotherapy (cisplatin and/or carboplatin) with or without irradiation between 2007-2014, and submitted to hearing monitoring before every cycle of chemotherapy and that completed a 5 years long-term follow-up. Chemotherapy regimens, demographic findings, cumulative doses and cranial irradiation were compared. Results. At the end of 5 -years follow-up ototoxicity was significantly increased compared to that at observed the end of chemotherapy $(52.5 \%$ vs $39.5 \%, \mathrm{p}<0.001)$. A late onset of hearing loss was experienced in $13.1 \%$ of children while in $26.3 \%$ progressive hearing loss was measured. Deafness at the end of chemotherapy and irradiation was significant prognostic factor for late ototoxicity outcomes (Odds Ratio 7.2 - CI:1.67-31.1 - p $<0.01$ and 5.25 - CI:1.26-21.86 - p $<0.01$ respectively). No significant differences were found between cisplatin and combined treatment (i.e. cisplatin shifted to carboplatin during monitoring for the onset of ototoxicity) and ototoxicity was not associated with platinum compounds cumulative dose ( $>>0.05)$. 13.1\% of children needed hearing aids at the end of follow-up. Conclusions. Our study confirms the effectiveness of long-term follow-up in identifying late onset/progressive hearing loss after platinum compound chemotherapy and allows us to avoid the harmful effects of hearing deprivation with hearing aid intervention.
\end{abstract}

Long-term auditory follow-up in counteracting platinum-induced ototoxicity in paediatric oncology

Anna R. Fetoni ${ }^{1}$, Francesca Brigato ${ }^{1}$, Eugenio De Corso $^{1}$, Daniela Lucidi $^{1}$, Walter Di Nardo ${ }^{1}$, Emanuele Scarano $^{1}$, Jacopo Galli ${ }^{1 \#}$, Antonio Ruggiero ${ }^{2 \#}$

${ }^{1}$ Department of Otolaryngology Head and Neck Surgery Fondazione Policlinico Universitario A. Gemelli IRCSS, Università Cattolica Sacro Cuore, 00168 Rome, Italy

${ }^{2}$ Pediatric Oncology Unit, Fondazione Policlinico Universitario A. Gemelli IRCCS, Università Cattolica Sacro Cuore, 00168 Rome, Italy

Word Count for:

a) Abstract: 245 words

b) Main Text: 3494 words

table:1 
Figures: 4

Running title: Long term auditory follow up in pediatric cancers

Keywords: ototoxicity; cisplatin; hearing aids; audiological monitoring; personalized medicine

\section{Corresponding Authors}

Anna R. Fetoni MD, PhD

Dept. of Otolaryngology Head and Neck Surgery

Università Cattolica Sacro Cuore,

Largo Francesco Vito 1, 000168 Roma, Italy

E-mail:annarita.fetoni@unicatt.it

Orcid \#: https://orcid.org/0000-0002-6955-7603

Eugenio De Corso MD, PhD

Dept. of Otolaryngology Head and Neck Surgery

Fondazione Policlinico A. Gemelli, IRCCS

Largo A. Gemelli, 800168 Roma, Italy

E mail: eugenio.decorso@policlinicogemelli.it

Table of abbreviations

\begin{tabular}{ll}
\hline dB HL & decibel hearing level \\
\hline ABR & Auditory Brain Response \\
SIOP & International Society of Pediatric Oncology \\
\hline
\end{tabular}

Abstract

Background. Irreversible bilateral sensorineural hearing loss is a common side effect of platinum compounds. Because of extended overall survival period of children, a prolonged hearing surveillance and management of hearing impairments are emerging concerns for pediatric oncology.

Methods. In this retrospective observational study we enrolled 38 children out of 116 treated at our institution by chemotherapy (cisplatin and/or carboplatin) with or without irradiation between 2007-2014, and submitted to hearing monitoring before every cycle of chemotherapy and that completed a 5 years long-term follow-up. Chemotherapy regimens, demographic findings, cumulative doses and cranial irradiation were compared.

Results. At the end of 5-years follow-up ototoxicity was significantly increased compared to that at observed the end of chemotherapy ( $52.5 \%$ vs $39.5 \%, \mathrm{p}<0.001$ ). A late onset of hearing loss was experienced in $13.1 \%$ of children while in $26.3 \%$ progressive hearing loss was measured. Deafness at the end of chemotherapy and irradiation was significant prognostic factor for late ototoxicity outcomes (Odds Ratio 7.2 - CI:1.67-31.1 - $\mathrm{p}<0.01$ and $5.25-\mathrm{CI}: 1.26-21.86-\mathrm{p}<0.01$ respectively). No significant differences were found between cisplatin and combined treatment (i.e. cisplatin shifted to carboplatin during monitoring for the onset of ototoxicity) and ototoxicity was not associated with platinum compounds cumulative dose ( $>0.05) .13 .1 \%$ of children needed hearing aids at the end of follow-up.

Conclusions. Our study confirms the effectiveness of long-term follow-up in identifying late onset/progressive hearing loss after platinum compound chemotherapy and allows us to avoid the harmful effects of hearing deprivation with hearing aid intervention. 


\section{Introduction}

The most common platinum-based agents, cisplatin and carboplatin, are widely used against several childhood and adolescent malignancies including neuroblastoma, osteosarcoma, germ cell tumours, teratoma, hepatoblastoma and a variety of brain tumours (e.g. glioma, astrocitoma, medulloblastoma) [1]. Dose depending sensorineural hearing loss is a common side effect of cisplatin chemotherapy in children. It is typically an irreversible bilateral hearing loss that initially involves high frequencies and with the progressive increase of the cisplatin cumulative dose also affects low frequencies. The prevalence of cisplatin-induced ototoxicity, measured with standard audiometry to $8 \mathrm{kHz}$, is approximately $60-70 \%$ in pediatric population $[2-4]$.

Although deafness is not a life-threatening condition, hearing loss can be detrimental for a child, especially on learning, academic performances and school behavior [5]. Deafness is associated with lower school functioning scores, greater need for special education services and poorer child-reported quality of life [6]. Furthermore, Authors demonstrated that, when hearing loss was serious enough to require hearing-aid use, it was independently associated with decline in cognition and educational performance $[7,8]$.

Unfortunately, the cochlear damage is irreversible, and involves various complex molecular mechanisms such us: induction of oxidative stress (i.e. reactive oxygen species production and lipid peroxidation), inflammation by activating pro-inflammatory factors and induction of p53-dependent signaling pathways $[9,10]$. It has been experimentally demonstrated that the outer hair cells are the major target for cisplatin ototoxicity, whereas carboplatin affects mainly the inner hair cells although it is considered less ototoxic than cisplatin [11-15]. Therefore, carboplatin may be introduced in children developing cisplatin ototoxicity [15]. In fact, it is possible to limit cisplatin cumulative dose damage by surveillance for hearing loss shifting the treatment by cisplatin to carboplatin at the onset of ototoxicity signs [15].

Implementation of the hospital protocols for auditory monitoring and the optimization of platinum-derived chemotherapy are challenging for children cancer treatment $[8,15]$ because effective preventive or curative therapeutic approaches in counteracting ototoxicity are lacking [14,16-22]. In fact, we demonstrated that the overall incidence of hearing loss was significantly decreased in children treated with cisplatin who underwent to auditory monitoring [Fetoni et al., 2016].

Thus, there is a general agreement on the role of surveillance across the clinical assessment of auditory function in order to early identify deafness during chemotherapy [23-28]. Nonetheless, a progressive worsening of hearing can appear several months after treatment even though it was normal at the end of treatment [29]. Therefore, a long-term follow-up can reveal not only a worsening of hearing loss in children who showed ototoxicity during treatment but also a late onset of hearing loss occurring months to years after therapy. How often and how long surveillance for hearing loss should be performed remains critical issues [29-31].

In this study we aimed to evaluate the risk of late ototoxicity both in terms of late new onset or progression in order to verify the hypothesis that long-term follow-up for ototoxicity is needed in children treated by platinum derived compounds chemotherapy. The early diagnosis in these patients is very important considering the lack of available otoprotectants, and requirement of an early rehabilitation by hearing aids, if needed, especially in children affected by comorbidities caused by their illness condition.

\section{Material and methods}

\section{Study population and study design.}

This is a retrospective observational study of children that underwent 5-years long-term hearing monitoring after chemotherapy and that were selected among 116 children treated with platinum compounds for solid tumours in our Institution from 2007 to 2014. The inclusion criteria were: age at the first platinum course [?]18 years, no administration of other ototoxic drugs, audiological baseline evaluation (within 2 weeks of 
the beginning of treatment), audiological monitoring during chemotherapy (including a re-evaluation prior to each chemotherapy cycle) and an yearly audiological long-term follow-up for at least 5 years after the end of chemotherapy. Exclusion criteria was a diagnosis of hearing loss at the baseline evaluation before chemotherapy.

Based on inclusion criteria we enrolled 38 children treated with platinum compounds and that completed a 5 years long-term follow-up. Patient's demographic characteristics, disease status and therapeutic strategies are summarized in Table 1. Briefly, median age at the beginning of treatments was 6 (range 1-18), the male-to-female ratio was 1,375. Most of the primitive lesions were brain tumours (glioma, medulloblastoma and astrocytoma), but aggressive tumours like neuroblastomas, osteosarcomas, germ cell tumours and retinoblastomas were also included.

About treatment modalities:

- Twenty-one patients (55.3\%) were treated with cisplatin with or without radiation therapy, of which $12(57.1 \%)$ switched to carboplatin because of toxicity onset, with a cisplatin's median cumulative dose of $245 \mathrm{mg} / \mathrm{sqm}$ (range 40-1080 mg/sqm) and six received a cumulative dose $>400 \mathrm{mg} / \mathrm{sqm}$;

- Seventeen patients $(44.7 \%)$ were treated with carboplatin with or without radiation therapy, with a median cumulative dose of $3400 \mathrm{mg} / \mathrm{m}^{2}$ (range $800-6050 \mathrm{mg} / \mathrm{m}^{2}$ ) of which one received a cumulative dose $>5000 \mathrm{mg} / \mathrm{m}^{2}$

Audiological monitoring.

Audiological monitoring was performed in our Institution by the same team of physicians and audiologists. At least a yearly evaluation was scheduled in patients with normal hearing whereas a six-monthly evaluation was performed in patients with a hearing loss diagnosis. Accordingly, with our previous paper [15], audiological evaluation included otoscopy and tympanometry and acoustic reflex measurements in order to exclude middle ear pathology (Grason Stadler, Eden Prairie Minn., USA).

Pure-tone air conduction thresholds were evaluated at frequencies of $0.25,0.5,1,2,4$ and $8 \mathrm{kHz}$ in decibel hearing level (dB HL). Pure-tone bone conduction thresholds were assessed at frequencies of $0.25,0.5,1,2$ and $4 \mathrm{kHz}$ to determine the type of the hearing impairment (i.e. conductive, sensorineural or mixed). Visual reinforcement audiometry was performed in a calibrated sound field or via earphones for children 6-30 months of age. Sound-field results reflect hearing in the better ear if there is a difference in hearing between ears. In general, frequencies at $0.5,1,2$ and $4 \mathrm{kHz}$ were obtained when visual reinforcement audiometry was used to measure hearing. Conditioned play audiometry (train show) was used for children able to cooperate (30 months to 5 years). Standard pure-tone audiometry was used for older children ( $>5$ years) under standard conditions using an Amplaid 319 audiometer (Amplaid Inc., Milan, Italy) in a double-walled, sound-proof room testing conventional frequency ranges from 0.25 to $8 \mathrm{kHz}$ (see www.asha.org/policy).

Auditory brain response (ABR) evaluation consisted of threshold searches using 100- $\mu$ s clicks as well as tone bursts at 1, 2 and $4 \mathrm{kHz}$ (GN Otometrics, Copenhagen, Denmark). Responses were obtained by averaged electroencephalic responses via three scalp electrodes positioned during sleep. We considered these examinations normal as their threshold was $20 \mathrm{~dB}$ HL bilaterally. Patients who had their baseline evaluations recorded by $\mathrm{ABR}$ where subsequently monitored by behavioural audiometry, and thus no patient underwent exclusive ABR evaluation.

Audiological records were examined and classified according to the SIOP (International Society of Pediatric Oncology) Boston ototoxicity scale (grade $0-4$ ). Families of children received adequate and comprehensible information about side effects of the administered therapy and scheduled audiological monitoring and gave their written consent to perform the tests. All protocol procedures were approved by the Ethical Committee of Fondazione Policlinico Universitario A. Gemelli IRCCS (Number of protocols: 0019971/20- ID 3074).

Statistical analyses.

Statistical analysis of data was performed through Microsoft EXCEL and IBM SPSS statistics 25 software 
package Armonk, NY IBM corp. Comparisons between groups were performed using the $\chi^{2}$ test, ANOVA test (Bonferroni) and Kruskal-Wallis test for independent samples. An uninominal logistic regression analyses was performed to analyze if prognostic factor hearing loss at the end of treatment was a significant prognostic factor for late ototoxicity outcomes. The results were considered significant with a p-value $<0.05$.

\section{Results}

Results at the end of chemotherapy and during the long-term follow-up.

Auditory assessment was performed in all children involved in this study before the beginning of chemotherapy and they showed normal hearing. Thirty-eight children completed the long-term audiological follow-up equal or longer than five years (range 60-108 months with a mean of 74 months). At the end of chemotherapy 15/38 (39.5\%) children presented ototoxicity (SIOP criteria $>0$ ), of which 10/38 (26.3\%) exhibited a progressive hearing loss during the long-term follow-up whereas $5 / 38(13.1 \%)$ did not progress furtherly hearing loss. In addiction at the long-term follow-up we observed 5/38 (13.1\%) new cases with late onset hearing loss. Finally, at the end of the 5-years follow-up 20/38 (52.5\%) had ototoxicity (SIOP criteria $>0$ ). No patient showed hearing improvement during the hearing monitoring. Details on grading percentages are showed in Fig. 1. The percentage of children with ototoxicity at the long-term follow-up was significantly increased compared to that at the end of chemotherapy $39.5 \%$ versus $52.5 \%(\mathrm{p}<0.001)$.

Sub-analyses on the late onset/progression cases.

Median time of the late onset/progression of ototoxicity was 24 months by the end of treatment (range: 3-84 months).

In the group of five children with a "late onset hearing loss" (Median age 10years; range 4-12; male/female ratio 4), four children showed a SIOP grade 1-2 and only one child a SIOP grade 4 at the end of 5-year followup. In this group four patient were exposed to cisplatin (no one with a cumulative dose $>400 \mathrm{mg} / \mathrm{sqm}$ ), and one to carboplatin therapy.

In the group of ten children (median age: 12.5 years; range: 2-18, male/female ratio was 2.33) that worsened over time a "progressive hearing loss", eight children developed a SIOP grade 4 and 2 children a SIOP grade 12. In this group five patients (50\%) were exposed to cisplatin plus carboplatin therapy (one with a cisplatin's cumulative dose $>400 \mathrm{mg} / \mathrm{sqm}$ ), one patient was exposed to cisplatin alone with a cumulative dose $>400$ $\mathrm{mg} / \mathrm{sqm}$, and four patients were exposed to carboplatin plus cranial irradiation therapy.

These results demonstrated that proportion of patients with SIOP grade 4 hearing loss was significantly higher in patients with hearing loss at the end of chemotherapy compared with patients with new late onset of ototoxicity, respectively 8/10 $(80 \%)$ versus $1 / 5(20 \%)(\mathrm{p}<0.05)$.

Finally, at the logistic regression analyses we observed that the presence of hearing loss at the end of treatment was a significant prognostic factor for long-term ototoxicity outcomes with an Odds Ratio of 7.2 (CI: 1.67-31.1; p < 0.01).

Prognostic factors of late ototoxicity outcomes.

According to our monitoring protocol, children treated with a second line carboplatin were those who shifted by cisplatin for the development of ototoxicity. Furthermore, children who underwent carboplatin therapy developed a SIOP grade 4 when subjected to prior or concurrent cranial irradiation for brain cancers. In fact, a significant difference $(\mathrm{p}=0.002)$ existed between children who underwent exclusive carboplatin therapy and those who were subjected to exclusive carboplatin and radiotherapy. Irradiation independently, if combined with cisplatin or carboplatin, was associated with a greater ototoxicity SIOP grade (Fig. 2) with a p-value of 0.001 . In addition, increasing age at treatment was correlated to greater hearing loss $(\mathrm{p}=0.013)$ as seen in Fig. 3, even if the older children underwent irradiation $(\mathrm{p}=0.012)$ as seen in Fig. 4. A severe late 
onset/progression ototoxicity was observed in 4/12 (33.3\%) of patients treated by cisplatin and carboplatin and in $1 / 8(12.5 \%)$ of patients treated by cisplatin alone $(\mathrm{p}>0.05)$.

The relationship between cumulative dose and incidence of hearing impairment has been analysed. Despite it is well known that ototoxicity is dose-dependent [15], in this cohort late onset/progression of hearing loss was not significantly associated with the cumulative dose of platinum compounds ( $\mathrm{p}>0.05)$. In addition, no significant differences have been observed between genders $(\mathrm{p}>0.05)$.

During follow-up, for five children (13.1\%), all of which developed a progressive hearing loss, audiological counselling was performed to inform family on hearing aids indication and support decision on hearing rehabilitation. In two aided children the progression of hearing loss occurred after 5 years of follow-up (i.e. 72 and 84 months after the end treatment). All of them were affected by medulloblastoma and were treated with platinum-derived compounds and brain irradiation therapy. Namely, three children were treated with cisplatin but for the onset of ototoxicity during audiological monitoring, shifted to carboplatin. Taken together, these data demonstrate that the early onset of ototoxicity during chemotherapy and concomitant brain irradiation significantly affect long-term hearing outcomes. At the logistic regression analyses we observed that the irradiation was a significant prognostic factor for long-term ototoxicity outcomes with an Odds Ratio of 5.25 (CI: $1.26-21.86 ; \mathrm{p}<0.01$ ).

\section{Discussion}

Nowadays, audiological monitoring is strongly recommended for ototoxicity surveillance [32] in pediatric oncology, especially if treated with platinum-derived compounds. In fact, it has been recently demonstrated, that the overall incidence of hearing loss can be significantly decreased by auditory monitoring during treatment because of early identification of ototoxicity and therapy shift to carboplatin [15]. Furthermore, late onset or progression of hearing loss after the end of therapy has been described $[7,8,29]$ even though few studies have been focused on long-term audiological monitoring. However, specific guidelines on long-term interventions in reducing late ototoxicity are still under discussion for health-care professionals in pediatric oncology.

Our aim was to provide evidence about the effectiveness of long-term surveillance in preventing platinuminduced hearing effects in children and adolescents surviving to cancer. In our experience, hearing status is currently measured after each cycle of cisplatin and adjustment of chemotherapy can be helped with suitable audiological counseling. When a child is diagnosed with grade $>2$ ototoxicity, cisplatin administration is stopped and changed to carboplatin. This protocol is able to achieve hearing loss diagnosis at an early stage and to provide, jointly with pediatric oncologists, therapeutic strategies to reduce further progression of ototoxic side effect shifting by cisplatin to carboplatin [15].

In our series we confirmed that hearing loss may develop and progress in children during platinum-based therapy [29], however deafness may increase over the years after the end of therapy. Furthermore, we interestingly demonstrated the high risk to develop a late onset/progressive hearing loss at 5-years follow-up $($ SIOP criteria $>0)$ that occurred in $15 / 38(39.5 \%)$ patients. These results are particularly important to identify cases requiring early hearing rehabilitative strategies to prevent harmful effect of hearing deprivation. In fact, we observed that $25 \%$ of monitored children needed hearing aid intervention.

How the ototoxicity appears or progresses over time is still unclear, nevertheless a prolonged maintenance of platinum in the body has been detected up to 20 years after treatment [33]. Furthermore, in experimental models, cisplatin has been demonstrated to target numerous cell types including outer hair cells and synapses between inner hair cells and terminal fibers of spiral ganglion neurons with a following neuronal degeneration that can appear at months after synaptic damage [10,34, 35]. Thus, as in the noise induced hearing loss and aging [36], the cisplatin-induced synaptopathy and neural degeneration might explain the compromised speech understanding in noisy environments especially observed in children that may lead to difficulties in school performances and psychosocial functioning [37, 38]. 
Platinum-induced hearing loss is irreversible [2, 15, 29], in fact in our experience no improvement of hearing was found over time, as expected we only observed a worsening of hearing after platinum therapy that was even more common than a late onset of hearing loss (i.e $26.3 \%$ vs $13.1 \%$ respectively). Among children having a progressive hearing loss, a SIOP grade 4 was found in 8/10 (80\%) indicating that the presence of hearing loss at the end of treatment was a negative prognostic factor for long-term ototoxicity outcomes (Odds Ratio: $7.2-$ CI: $1.67-31.1 ; \mathrm{p}<0.01$ ). Furthermore, median time of the late onset and progression of ototoxicity was 24 and 18 months respectively by the end of treatment. The early onset of ototoxicity as detected by the auditory monitoring is suggestive for a worse audiological outcome over time likely depending by individual susceptibility that is still understudied. A genetic predisposition has been proposed based upon observations of substantial inter-individual variability in the prevalence and severity of ototoxicity including polymorphisms for genes involved in the oxidative stress, which is the major target of cisplatin ototoxicity [15, 39]. Unfortunately, a routinely analysis of genetic polymorphism is still lacking [40], therefore the long-term audiological monitoring remains a major target for prevention of ototoxicity.

Traditionally, cumulative doses, age at treatment, cranial irradiation and type of malignances have been considered as risk factors for platinum-induced ototoxicity in pediatric population [40, 41]. In contrast to previous findings [43], we found that the cumulative dose of cisplatin, affecting the early onset of hearing loss [15], did not correlate with long-term development of ototoxicity.

Remarkably, we found that the risk of hearing loss was high associated with cisplatin-based multimodal therapy that included carboplatin and/or radiotherapy rather than a cumulative dose of cisplatin alone that is currently suggested as indicator for hearing screening. Brain irradiation is then an independent factor for the occurrence of ototoxicity during platinum chemotherapy [42, 43]. In fact, most of children treated with carboplatin were those who developed ototoxicity during cisplatin treatment and then shifted to less ototoxic carboplatin; probably susceptibility to cisplatin damage remains major trigger for development of hearing loss. Furthermore, children who underwent carboplatin therapy alone developed a SIOP grade 4 when subjected to prior or concurrent cranial irradiation for brain cancers. Therefore, irradiation remains even in our experience a prognostic factor for long-term ototoxicity outcomes (Odds Ratio of 5.25 - CI: $1.26-21.86 ; \mathrm{p}<0.01)$.

Hence, in the pre-treatment counseling, it is important for pediatric oncologist to inform families on the possibility to change chemotherapy avoiding ototoxicity during treatment and indication for a long-term audiologic follow-up, especially in children affected by brain cancers treated with radiotherapy. In these cases, whether shifting by cisplatin to carboplatin the overall survival can be affected is a controversial issue that needs to be further discussed with family [1].

We found a worse hearing in older children, conversely in literature a higher ototoxicity is reported in younger age $[4,32,41,43]$. In our study, although the limitation in size sample, older children were affected by medulloblastoma, which is even a rare cancer, and experienced a worse hearing loss. This could be explained as the most of them was treated with both platinum compound and irradiation therapy. While, younger children affected by neuroblastoma and retinoblastoma were mainly treated with carboplatin.

A great variability of ototoxic outcomes has been reported [41, 43] that might depend on differences in assessment strategies, such as inclusion criteria, patient population, methodological techniques, auditory measurements and duration of follow-up. Worthy of note, among different scales we use the Boston SIOP ototoxicity scale, which is sensitive to high frequencies of hearing, the most affected by platinum-derived ototoxicity, even if in younger children they are more difficult to detect $[32,41$,$] . Probably there is not$ a comprehensive scale for all conditions considering the variability of audiological assessment for age at treatment, status of illness of children during testing, concomitant cognitive and sensorial impairment. However, this grading system seems to be able to include a larger number of affected children.

In our series, five out of children who progressed hearing at the long-term follow-up required hearing aid rehabilitation. Deafness, especially during childhood and even when only borderline to mild, can have important implications. Therefore, a parental counselling on auditory diagnosis and benefits of hearing aids 
is recommended. The early and appropriate hearing rehabilitation that better suits children needed is crucial for brain plasticity maintenance during sensitive period and language development, social integration [44] and quality of life considering that most of these are multi-handicap patients, mainly with sensory deprivation (i.e. visual) that further affects outcomes. While in teenager the high-frequency hearing loss can reduce speech recognition in noisy environment affecting learning and communicative abilities, especially during school performances. Thus, hearing aids and the use of assistive listening devices such as frequency modulation systems are suitable. Additionally, audiological surveillance is useful in children affected by central nervous system tumours since hearing loss is associated with worse physical well-being, poorer relationship with peers and greater need for social support compared to normal hearing [45]. Furthermore, most of survival children live far from tertiary oncologic institutions, therefore many years after the end of therapies oncological check-ups become less frequent; many families continue their sporadic radiologic and clinical controls at their hometown hospital, making it difficult to obtain a long-term reliable hearing assessment.

Parents may be advocated by the supporting services so that their child is not lost to follow-up once they enter survivorship. In addition to monitoring hearing provides beneficial effects on children and young adults in understanding their hearing status, its impact on communication and learning, hearing conservation, and their options for ongoing management.

In conclusion, platinum compounds can lead to bilateral, late onset or progressive and permanent sensorineural hearing loss affecting language development. Therefore, long-term monitoring is recommended in all children considering that deafness is dependent by treatments and individual susceptibility that likely trigger the onset and progression of ototoxicity. To date, the long-term monitoring at least of 5 years, especially in children who underwent multimodal therapy including irradiation, may offer the highest level of care to our patients and their families improving quality of life after successful oncological treatment.

\section{Conflict of Interest}

The authors declare that the research was conducted in the absence of any commercial or financial relationships that could be construed as a potential conflict of interest.

\section{Acknowledgments}

The authors express their gratitude to Bianca Maria Martina, Paola Torraco, Francesco Minioto and Mauro De Carlini for their helpful contribution and technical assistance in audiological assessments

\section{References.}

[1]. Ruggiero A, Trombatore S, Triarico R, Arena R, Ferrara M, Scalzone M, Pierri F, Riccardi R. Platinum compounds in children with cancer: toxicity and clinical management. Anti Canc Drugs 2013; 24. 1007-1019

[2]. Knight KR, Kraemer DF, Neuwelt, EA. Ototoxicity in children receiving platinum chemotherapy: underestimating a commonly occurring toxicity that may influence academic and social development. J Clin Oncol 2012;23:8588-8596

[3]. Bass JK, Huang J, Onar-Thomas A, Chang K W, Bhagat SP, Chintagumpala M, Bartels U, Gururangan S, Hassall T, Heath JA, McCowage G, Cohn RJ, Fisher MJ, Robinson G, Broniscer A, Gajjar A, Gurney JG. Concordance between the Chang and the International Society of Pediatric Oncology (SIOP) ototoxicity grading scales in patients treated with cisplatin for medulloblastoma. Pediat Blood Cancer (2014); 61:601605 
[4]. Peleva E., Emami N., Alzahrani M., Bezdjian A., Gurberg J., Carret AS, Daniel SJ. Incidence of platinum-induced ototoxicity in pediatric patients in Quebec. Pediatr Blood Cancer 2104; 61:2012-2017

[5]. Davis J M, Elfenbein J, Schum R, Bentler R A. Effects of mild and moderate hearing impairments on language, educational, and psychosocial behaviour of children. J Speech Hear Disord 1986; 51:53-62

[6]. Gurney J.G., Tersak J.M., Ness K.K., Landier W., Matthay K.K., Schmidt ML, Children's Oncology Group. Hearing loss, quality of life, and academic problems in long-term neuroblastoma survivors: a report from the Children's Oncology Group. Pediatrics 2007; 120:e1229-1236

[7]. Schreiber JE, Gurney JG, Palmer SL, Bass J K., Wang M, Chen S, Zhang H, Swain M, Chapieski ML, Bonner MJ:, Mabbott DJ. Knight SJ., Armstrong CL, Boyle B, Gajjar A. Examination of risk factors for intellectual and academic outcomes following treatment for paediatric medulloblastoma. Neuro Oncol 2014; 16:1129-1136

[8]. Brooks B, Knight K. Ototoxicity monitoring in children treated with platinum chemotherapy. Int J Audiol 2018; 57:S34-S40

[9]. Fetoni AR, Paciello F, Rolesi R, Paludetti G, Troiani D. Targeting dysregulation of redox homeostasis in noise-induced hearing loss: Oxidative stress and ROS signaling. Free Radic Biol Med 2019; 135:46-59

[10]. Paciello F, Fetoni A R, Mezzogori D, Rolesi R, Di Pino A, Paludetti G, Grassi C, Troiani D. The dual role of curcumin and ferulic acid in counteracting chemoresistance and cisplatin-induced ototoxicity. Sci Rep 2020; 10:1063-1067

[11]. Takeno S, Harrison RV, Ibrahim D, Wake M, Mount RJ.Cochlear function after selective inner hair cell degeneration induced by carboplatin. Hear Res 1994;75:93-102

[12]. Punnett A, Bliss B, Dupuis LL, Abolell M, Doyle J, Sung L. Ototoxicity following pediatric hematopoietic stem cell transplantation: a prospective cohort study. Pediatr Blood Cancer (2014) 42:598-603

[13]. Qaddoumi I, Bass J K, Wu J, Billups CA, Wozniak AW, Merchant TE, Haik BG, Wilson MW, Rodriguez-Galindo C. Carboplatin-associated ototoxicity in children with retinoblastoma. J Clin Oncol 2012; 30:1034-1041

[14]. Fetoni AR, Paciello F, Mezzogori D, Rolesi R, Eramo SL, Paludetti G, Troiani D. Molecular targets for anticancer redox chemotherapy and cisplatin-induced ototoxicity: the role of curcumin on pSTAT3 and Nrf-2 signalling. Br J Cancer 2015; 113:1434-1444

[15]. Fetoni A R, Ruggiero A, Lucidi D, De Corso E, Sergi B, Conti G, Paludetti G. Audiological monitoring in children treated with platinum chemotherapy. Audiol Neurootol 2016; 21:203-211

[16]. Kalkanis JG, Whitworth C, Rybak LP. Vitamin E reduces cisplatin ototoxicity. Laryngoscope 2004; $114: 538-542$

[17]. Choe W. T., Chinosornvatana N., Chang K. W. (2004), Prevention of cisplatin ototoxicity using transtympanic N-acetylcysteine and lactate, Otol. Neurotol., 25:910-915

[18]. Grewal S, Merchant T, Reymond R, Hodge C M, Shearer P. Auditory late effects of childhood cancer therapy: A report from the Children's Oncology Group. Pediatrics 2010; 125:e938-950

[19]. Olgun Y, Kırkım G, Kolatan E, Kıray M, Bagriyanık A, Olgun A, Kızmazoglu DC, Ellıdokuz H, Serbetcioglu B, Altun Z, Aktas S, Yılmaz O, Günerı EA. Friend or foe? Effect of oral resveratrol on cisplatin ototoxicity. Laryngoscope 2014; 124:760-766

[20]. Hughes L, Hussain N, Pafford R, Parham K. Dexamethasone otoprotection in a multidose cisplatin ototoxicity mouse model. Otolaryngol Head Neck Surg 2014; 150:115-120 
[21]. Marshak T, Steiner M., KaminerM, Levy A, Shupak A. Prevention of cisplatin-induced hearing loss by intratympanic dexamethasone: a randomized controlled study. Otolaryngol Head Neck Surg 2014; 50:983990

[22]. Laurell G. Pharmacological intervention in the field of ototoxicity. HNO 2019; 67:434-439

[23]. Audiologic management of individuals receiving cochleotoxic drug therapy. Guidelines developed by the American Speech-Language-Hearing Association (ASHA), 1994; 36(supplement 12):11-19

[24]. Stavroulaki P, Apostolopoulos N, Segas J, Tsakanikos M, Adamopoulos G. Evoked otoacoustic emissions-an approach for monitoring cisplatin induced ototoxicity in children, Int J Pediatr Otorhinolaryngol 2011; 59:47-57

[25]. Romano A, Capozza MA, Mastrangelo S. Maurizi P, Triarico S, Rolesi R, Attinà G, Fetoni AR, Ruggiero A. Assessment and management of platinum-related ototoxicity in children treated for cancer, Cancers (Basel), 2020; 2:E1266

[26]. Konrad-Martin D, Helt DJ, Reavis KM, Ototoxicity: early detection and monitoring. ASHA Leader $2004 ; 10: 1-14$

[27]. Jacob BCB, Aguiar FP, Tomiasi AA, Tschoeke SN, De Bitencourt RF. Auditory monitoring in ototoxicity. Braz J of Otorhinolaryngol 2006; 72:836-844

[28]. Nitz A, Kontopantelis E, Bielack S, Koscielniak E, Klingebiel T, Langer T, Paulides M, Prospective evaluation of cisplatin- and carboplatin-mediated ototoxicity in paediatric and adult soft tissue and osteosarcoma patients. Oncol Lett 2013;5:311-315

[29]. Bertolini P, Lassalle M, Mercier M, Raquin MA, Izzi G, Corradini N, Hartmann O. Platinum compoundrelated ototoxicity in children: long-term follow-up reveals continuous worsening of hearing loss. J Pediatr Hematol Oncol 2004; 26:649-655

[30]. Al-Khatib T, Cohen N, Carret AS, Daniel S. Cisplatinum ototoxicity in children, long-term follow-up. Int J Pediatr Otorhinolaryngol 2010; 74:913-919

[31].Einarsson EJ, Petersen H, Wiebe T, et al. (2010), Long-term hearing degeneration after platinum-based chemotherapy in childhood, Int J Audiol 2010; 49:765-771

[32]. Clemens E, van den Heuvel-Eibrink MM, Mulder RL, Kremer LCM, Hudson MM, Skinner R, Constine LS, Bass LK, Kuehni CE, Langer T, C van Dalen E, Bardi E, Bonne NX, Brock PR, Brooks B, Carleton B, Caron E, International Guideline Harmonization Group ototoxicity group. International Guideline Harmonization Group ototoxicity group. Recommendations for ototoxicity surveillance for childhood, adolescent, and young adult cancer survivors: a report from the International Late Effects of Childhood Cancer Guideline Harmonization Group in collaboration with the PanCare Consortium. Lancet Oncol 2019; 20:e29-41

[33]. Gietema JA, Meinardi MT, Messerschmidt J, Gelevert T, Alt F, Uges DR, Sleijfer DT. Circulating plasma platinum more than 10 years after cisplatin treatment for testicular cancer. Lancet 2000; $25 ; 355(9209): 1075-6$

[34].Lu J, Wang W, Liu H, Wu H. Cisplatin induces calcium ion accumulation and hearing loss by causing functional alterations in calcium channels and exocytosis. Am J Transl Res 2019; 11:6877-6889

[35]. Sheth S, Sheehan K, Dhukhwa A, Al Aameri RFH, Mamillapalli C, Mukherjea D, Rybak LP, Ramkumar V. Oral administration of caffeine exacerbates cisplatin-induced hearing loss. Sci Rep 2019; 9:9571-95

[36]. Liberman MC, Kujawa SG. Cochlear synaptopathy in acquired sensorineural hearing loss: manifestations and mechanisms. Hear Res 2017;349:138-147

[37]. Skinner R. Best practice in assessing ototoxicity in children with cancer. Eur J Cancer 2004; 40:2352-4 
[38]. Dean JB, Hayashi SS, Albert CM, King AA, Karzon R, Hayashi RJ. Hearing loss in pediatric oncology patients receiving carboplatin-containing regimens. J Pediatr Hematol Oncol 2008; 30:130-4

[39]. Tserga E, Nandwani T, Edvall NK, Bulla J, Patel P, CanlonB, Cederroth CR, Baguley DM. The genetic vulnerability to cisplatin ototoxicity: a systematic review. Sci Rep 2019; 9:3455, doi: 10.1038/s41598-01940138-z

[40]. Waissbluth S, Chuang A, Del Valle A, Cordova M. Long-term platinum-induced ototoxicity in pediatric patients. Int J Pediatr Otorhinolaryngol 2018;107:75-79

[41]. Wei M, Yuan X. Cisplatin-induced ototoxicity in children with solid tumor. J Pediatr Hematol Oncol 2019; 41:e97-e100

[42]. Hamilton SH, Arshad O, Kwok J, Tran E, Howard AF, Serrano I, Goddard K. Documentation and incidence of late effects and screening recommendations for adolescent and young adult head and neck cancer survivors treated with radiotherapy. Supp Care Cancer 2019; 27:260 9-2616

[43]. Clemens E, Brooks B, de Vries ACH, van Grotel M, van den Heuvel-Eibrink MM,Carleton B. A comparison of the Muenster, SIOP Boston, Brock, Chang and CTCAEv4.03 ototoxicity grading scales applied to 3,799 audiograms of childhood cancer patients treated with platinum-based chemotherapy. PLoS One 2019;14:e210646

[44]. Kral A, Dorman MF, Wilson BS. Neuronal development of hearing and language: cochlear implants and critical periods. Ann Rev Neurosci 2019; 42:47-65

[45]. Weiss A, Sommer G, Schindera C, Wengenroth L, KarowA, Diezi M, Michel G, Kuehni EC, Swiss Paediatric Oncology Group (SPOG). Hearing loss and quality of life in survivors of paediatric CNS tumours and other cancers. Qual Life Res 2019; 28:515-521

\section{Legends.}

TABLE 1 Patients' demographic characteristics and treatments

FIGURE 1: SIOP grading at the end of the therapy and at 5-years follow-up

FIGURE 2: Differences in ototoxicity SIOP grade in children who were subjected to radiotherapy and who were not.

FIGURE 3: Distribution of hearing loss according to the age

FIGURE 4: Distribution of radiotherapy according to the age

\section{Hosted file}

table 1.pdf available at https://authorea.com/users/364446/articles/484862-long-termauditory-follow-up-in-counteracting-platinum-induced-ototoxicity-in-paediatric-oncology 

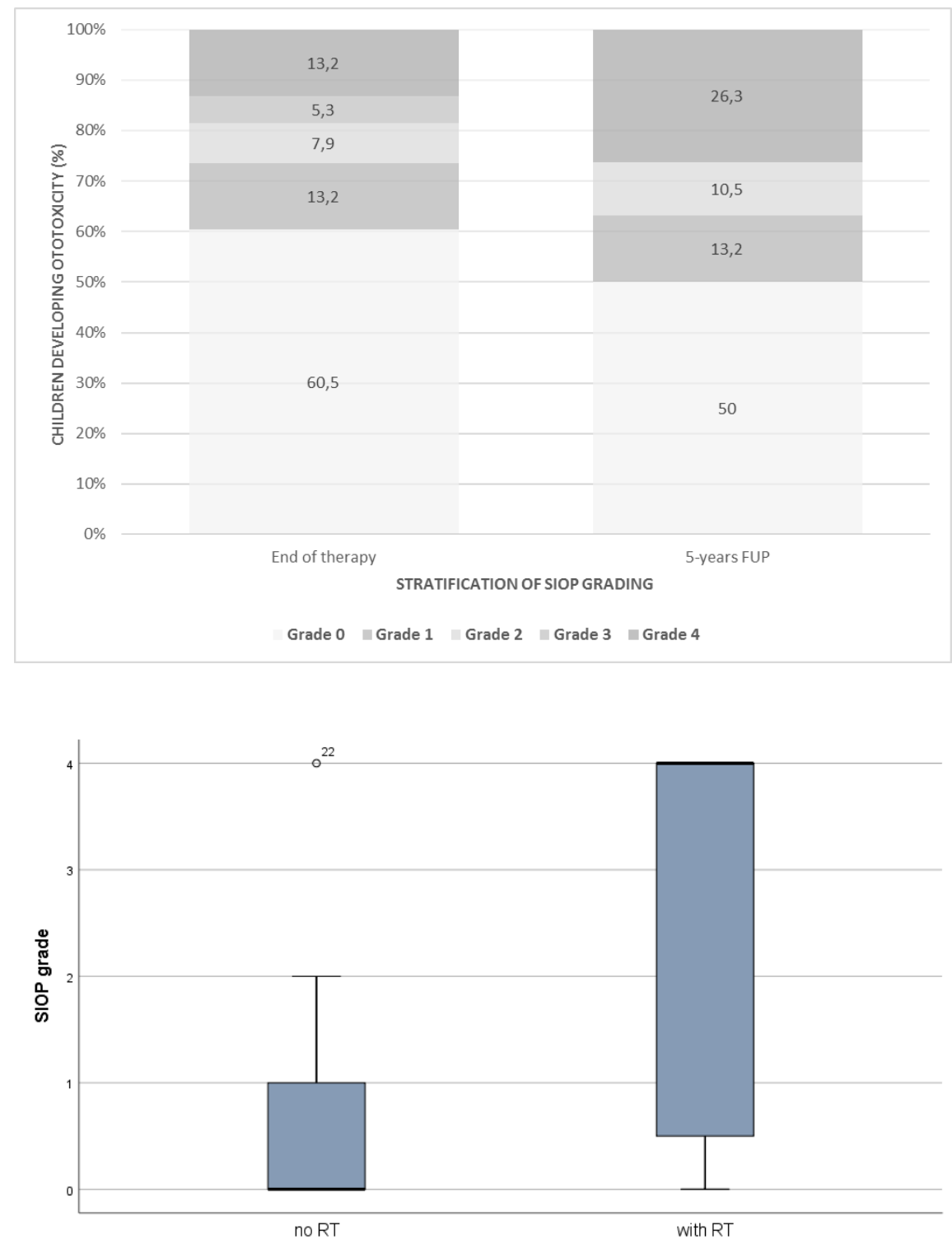

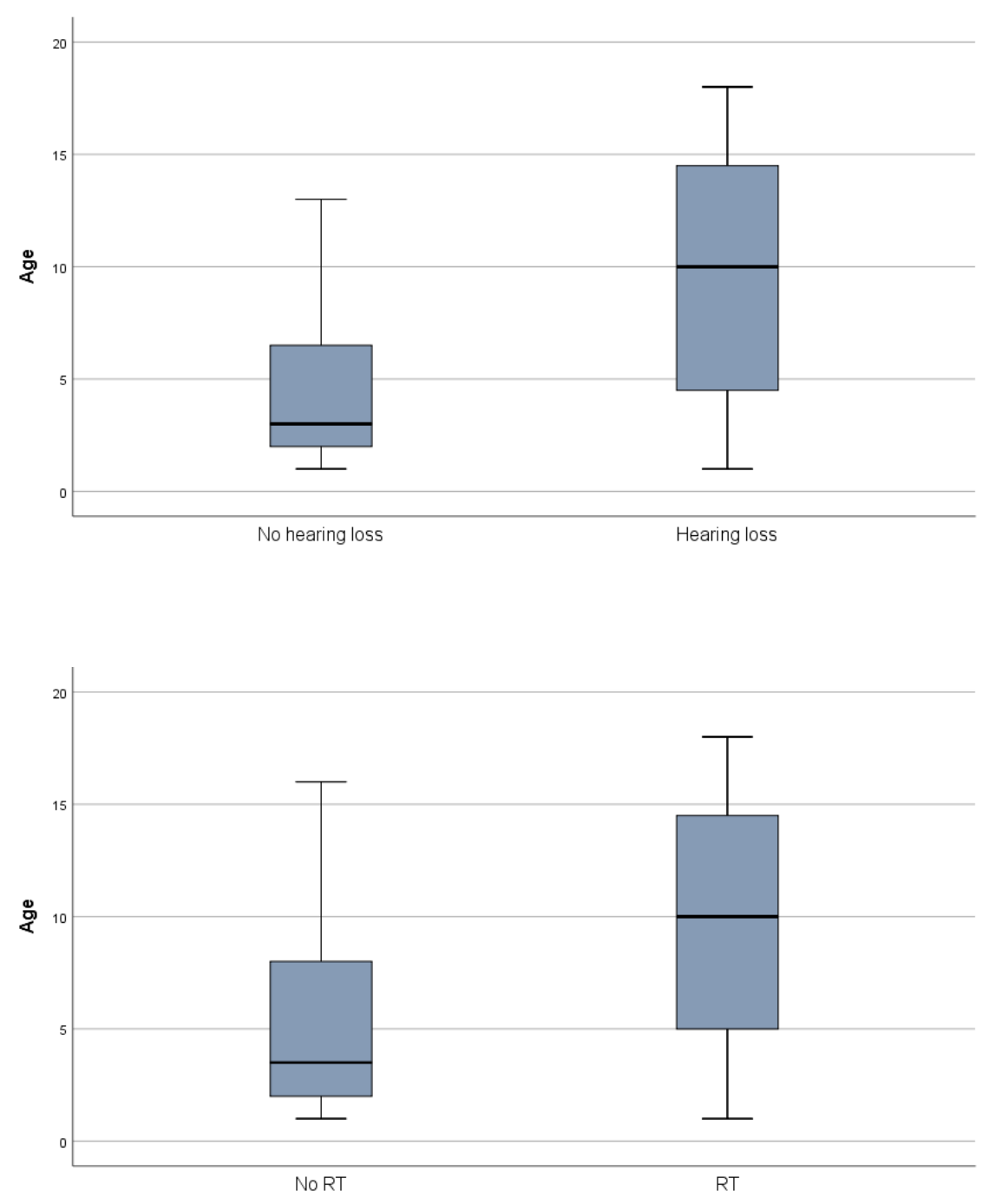